\title{
Relativistic description of astronomical objects in multiple reference systems
}

\author{
Chongming $\mathrm{Xu}^{1} \&$ Zhenghong Tang ${ }^{2}$ \\ Shanghai Astronomical Observatory, Chinese Academy of Sciences, P.R.China; \\ email: ${ }^{1}$ cmxu1938@gmail.com, ${ }^{2}$ zhtang@shao.ac.cn
}

\begin{abstract}
Many astronomical systems require for their description in the frame of Einstein's theory of gravity not just one but several reference systems. In the first post-Newtonian approximation the Damour-Soffel-Xu (DSX) formalism presents a new and improved treatment of celestial mechanics and astronomical reference systems for the gravitational $N$-body problem. In the DSX-formalism the astronomical bodies are characterized by their Blanchet-Damour (BD) mass- and spin-multipole moments. However, the time dependence of these moments requires additional dynamical equations, usually local flow equations describing the internal motions inside the bodies or additional assumptions about them. In this article the internal motion of astronomical bodies will be adressed within the 1st post-Newtonian approximation to Einstein's theory of gravity. A concept of quasi-rigid bodies will be introduced; after that, astronomical fluid and elastic bodies will be discussed.
\end{abstract}

Keywords. GRT, relativistic description of astronomical objects, post-Newtonian approximation

\section{Introduction}

We are at the point where astronomical measurements will soon reach incredible accuracies; e.g., the astrometric satellite Gaia will measure angular distances with a precision of about $10 \mu \mathrm{as}$. At this level of accuracy not only the various astronomical reference systems, but also the internal motion of astronomical bodies have to be described in the framework of relativity, at least at the first post-Newtonian approximation. The problem of relativistic astronomical reference systems and the applications of the standard IAU framework have been discussed by Soffel (2009) in detail. In this paper we discuss the problem of internal motion of astronomical bodies. The discussion will be such that several reference systems should be used to describe the motion of the whole system relativistically assuming e.g., that the astronomical body is member of an $N$-body system. A (DSX) framework dealing with the celestial mechanics of $N$ rotating bodies of arbitrary shape and composition at the first post-Newtonian approximation to Einstein's theory of gravity has recently been introduced by Damour, Soffel \& Xu (1991, 1992, 1993, 1994). This framework employs a total of $N+1$ different coordinate (reference) systems: one global one that covers the entire model manifold to describe the motion of the whole $N$-body system and one local system co-moving with each of the $N$ bodies to describe the local physics, especially the internal motion of the body under consideration. In the DSX-framework the gravitational potentials (one scalar and one vector potential) of some body in the outside region is described by a set of mass- and a set of spin-multipole moments (Blanchet-Damour moments) in the corresponding local comoving system. If $(c T, \boldsymbol{X})$ denote the coordinates of some local A-system these BD-moments are defined 
by

$$
\begin{aligned}
M_{L}^{A}(T) \equiv & \int_{A} d^{3} X \hat{X}^{L} \Sigma+\frac{1}{2(2 l+3) c^{2}} \frac{d^{2}}{d T^{2}}\left[\int_{A} d^{3} X X^{L} X^{2} \Sigma\right] \\
& -\frac{4(2 l+1)}{(i+1)(2 l+3) c^{2}} \frac{d}{d T}\left[\int_{A} d^{3} X X^{a L} \Sigma^{a}\right]+O(4) \quad(l \geqslant 0), \\
S_{L}^{A}(T) \equiv & \int_{A} d^{3} X \epsilon^{a b<c_{l}} \hat{X}^{L-1>a} \Sigma^{b}+O(2) \quad(l \geqslant 1),
\end{aligned}
$$

where $L$ is a multi-index of $l$ Cartesian indices: $L=i_{1} i_{2} \cdots i_{l}$; both, the mass moments $M_{L}$ and the spin moments $S_{L}$ are assumed to be symmetric and trace-free (STF); $\Sigma$ and $\Sigma^{a}$ are the active gravitational mass and active mass current density which are defined as $\Sigma=c^{-2}\left(T^{00}+T^{b b}\right)$ and $\Sigma^{a}=c^{-1} T^{0 a}$ in the local coordinate system. $T^{\alpha \beta}$ are the contravariant components of the stress-energy tensor. The order symbol, $O(n)$, in the equation indicates that terms of order $c^{-n}$ have been neglected.

The two gravitational potentials, $W_{A}$ and $W_{A}^{a}$, appearing in the canonical form of the local metric tensor (e.g., Damour, Soffel \& Xu 1991) outside of body $A$ can easily be expressed in terms of the BD moments of $A$. For astronomical bodies of almost spherical shape this expansion will converge rapidly.

In the DSX-framework the time dependence of the BD-moments has not been treated in detail. It depends upon the internal dynamics of the astronomical body, which is the main topic of this article.

For many astronomical bodies (e.g., stars) it is sufficient to assume the internal material to be fluid. Corresponding Newtonian hydrodynamical and thermodynamical equations can be found in many textbooks. Relativistic hydrodynamical and thermodynamical equations have been discussed, e.g., by Chandrasekhar (1971), who, however, employs a single coordinate system. Wu and Xu (2001) have discussed such equations in the problem of multiple reference systems which might be relevant for close binary or multiple stellar systems.

For other astronomical bodies, such as the Earth or neutron stars, the elastic components cannot be described by a fluid picture. Here, the formalism of relativistic elastomechanics can be employed. Within the DSX-framework such a formalism has been worked out by Xu, Wu \& Soffel (2001) and Xu et al., (2003, 2005). This formalism is based on a displacement-field as is the ell known Newtonian formalism of elastomechanics. For applications in the field of geodynamics post-Newtonian equations for the displacement field have been derived that generalize the well known Jeffreys-Vicente (Jeffreys \& Vicence 1957) equations from Newtonian physics.

Both, the fluid and elastic material of astronomical bodies will be treated below. In Newtonian celestial mechanics, however, dynamical equations are often drastically simplified by assuming the astronomical bodies to be rigid (only three internal degrees of freedom). For the construction of a solar system ephemeris such an assumption is a good starting point. E.g., for the problem of Earth rotation in the Newtonian framework the well known SMART solution (Bretagnon et al., 1997), which might serve as excellent basis for treating the real problem of geodynamics, is based on 'rigid bodies'. It is well known that rigid bodies with incompressible material elements do not exist in relativity since in reality the sound speed always has to be finite. Nevertheless, quasi-rigid bodies may be introduced by auxiliary conditions that simplify the post-Newtonian formalism drastically. Such quasi-rigid relativistic bodies have been treated in Xu \& Tao (2004) and Tao \& Xu (2003). 


\section{Quasi-rigid bodies}

For our model of a quasi-rigid astronomical body a certain relation for the internal motion is formulated in the local system that is co-moving with the body under consideration. We can define the quantities $\bar{\Sigma}$ and $\overline{\Sigma^{a}}$ by

$$
\begin{aligned}
\bar{\Sigma} & \equiv \Sigma+\frac{\Sigma_{\mathrm{PN}}}{c^{2}}, \quad \text { where } \Sigma_{\mathrm{PN}} \equiv \frac{11}{42} X^{2} \ddot{\Sigma}, \\
\bar{\Sigma}^{a} & \equiv \Sigma^{a}+\frac{\Sigma_{\text {self }}^{a}}{c^{2}}+\frac{\Sigma_{\text {ext }}^{c}}{c^{2}}, \\
\Sigma_{\text {self }}^{a} & \equiv \Sigma\left(\frac{7}{2} \epsilon_{a d e} \Omega^{d} \partial_{e} Z^{+}+\frac{1}{2} \epsilon_{e d f} \Omega^{d} X^{f} \partial_{a e} Z^{+}\right), \\
Z^{+} & \equiv G \int_{A} d^{3} X^{\prime} \Sigma\left(T_{A}, \mathbf{X}^{\prime}\right)\left|\mathbf{X}-\mathbf{X}^{\prime}\right|, \\
\Sigma_{\text {ext }}^{a} & \equiv \sum_{l \geqslant 0} \frac{\Sigma}{l !}\left[4 \epsilon_{a d e} \Omega^{d} X^{e} X^{<L>} G_{L}(T)+\frac{1}{l+2} \epsilon_{a e d} X^{<d L>} H_{e L}\right. \\
& \left.-\frac{l+10}{2(l+2)(2 l+5)} \hat{X}^{L} X^{2} \dot{G}_{c L}+\frac{l+10}{2(l+2)(2 l+5)} \partial_{T}(\ln \Sigma) \hat{X}^{L} X^{2} G_{c L}\right],
\end{aligned}
$$

where $G_{L}$ and $H_{L}$ are gravito-electric and gravito-magnetic tidal moments (e.g., Damour, Soffel \& Xu 1991). Now, a model of a 1PN quasi-rigid body can be constructed by means of the following constraint:

$$
\bar{\Sigma}^{a}+\frac{1}{2 c^{2}} X^{a}\left[\partial_{T} T^{b b}-\Sigma \partial_{T} W+\partial_{T} \Sigma_{\mathrm{PN}}+\partial_{a}\left(\Sigma_{\text {self }}^{a}+\Sigma_{\text {ext }}^{a}\right)\right]=\epsilon_{a b c} \Omega^{b} X^{c} \bar{\Sigma}+O(4) .
$$

In this equation $\Omega^{a}$ might be considered as a formal parameter of our quasi-rigid body, whereas in the Newtonian approximation $\Omega^{a}$ is the angular velocity. Our constraint equation has the following consequences: The 1PN spin vector (defined in Damour, Soffel \& $\mathrm{Xu} 1993)$ is given by

$$
S_{a}^{\mathrm{PN}}=I_{a b} \Omega^{b}+O(4)
$$

where the $1 \mathrm{PN}$ moment of inertia tensor reads

$$
I_{a b}=I_{b a}=\int_{A} d^{3} X\left(\delta_{a b} \mathbf{X}^{2}-X^{a} X^{b}\right) \bar{\Sigma}+O(4) .
$$

The post-Newtonian MacCullagh relations then simply read

$$
M_{a b}=-I_{a b}+\frac{1}{3} \delta_{a b} I_{c c}+O(4) .
$$

As is well known such MacCullagh relations between components of the inertia tensor and potential coefficients are very useful in geodynamics. Actually, with our constraint equation all relations for 'Newtonian rigid bodies' are valid even at the first post-Newtonian level except for one:

$$
\dot{M}_{L}=\epsilon_{p q<a_{l}} M_{L-1>q} \Omega^{p}, \quad l>2 .
$$

This difference, however, might not be relevant in most situations since the relativistic part of $M_{L}$ with $L>2$ usually can be neglected.

\section{Elastic and fluid bodies}

Our formalism that describes the dynamics of elastic material based upon a displacement field presents an application of the Carter and Quintana (Carter \& Quintana 1972) 
formalism. Let $s^{a}$ denote the components of the displacement field that describes deviations from an equilibrium configuration (the elastomechanical ground state). The postNewtonian Jeffreys-Vicente ( $\mathrm{J}-\mathrm{V})$ equation for an almost spherical body then reads $(\mathrm{Xu}$, Wu \& Soffel 2001):

$$
\begin{aligned}
\rho^{*} \frac{D^{2} s_{a}}{D \tau^{2}}= & \bar{W}_{G, a} \delta \rho^{*}+\rho^{*}(\delta \bar{W})_{, a}-\frac{1}{2} \rho^{*}\left(\bar{V}^{2}\right)_{, a b} \dot{s}^{b}-(\delta p)_{, a}+\left(2 \mu s_{a}^{b}\right)_{; b} \\
& +\frac{1}{c^{2}}\left\{\rho ^ { * } \left[-\frac{1}{2}\left(\bar{V}^{2}\right)_{, a b} s^{b} \bar{V}^{2}-\bar{V}^{a}\left(\bar{V}^{b} \ddot{s}^{b}\right)+4 W_{\mathrm{G},[a} \bar{V}^{b]} \dot{s}^{b}-(\delta \bar{W})_{, \bar{T}} \bar{V}^{a}\right.\right. \\
& \left.\left.+4\left(\delta \bar{W}_{a}\right)_{, \bar{T}}+\bar{W}_{, a} \bar{W}_{, b} s^{b}-8 \bar{V}_{,(c}^{b} \bar{W}_{[a), b]} s^{c}\right]-\delta \dot{p} \bar{V}^{a}\right\}+O(4),
\end{aligned}
$$

where the variables with a bar are in rotating coordinates (angular velocity: $\Omega^{a}$ ), $\rho$ is the mass-energy density $\left(\rho^{*}=\rho+p c^{-2}\right), \mu$ denotes the shear modulus, $\bar{W}_{G}$ is the PN geopotential $\bar{W}_{G}=\bar{W}+\frac{1}{2} \bar{V}^{2}, \bar{V}^{a}$ the rotation velocity, $\bar{W}$ and $\bar{W}^{a}$ the scalar potential and vector potential of the metric tensor; $\delta p, \delta \rho, \delta W$ are the Eulerian variation of $p, \rho$ and $W$, respectively. For $c \rightarrow \infty$, the above equation reduces to the Newtonan J-V equation. Boundary and junction conditions of this relativistic J-V equation have been discussed in $\mathrm{Xu}$ et al. (2003). The PN J-V equation and related equations have been expanded in terms of generalized spherical harmonics so that the partial differential equations become a set of ordinary differential equations (Xu et al., 2005) to simplify further calculations.

A fluid body might be viewed as a special case of an elastic body. Corresponding hydrodynamical equations have been discussed by $\mathrm{Xu}$ and $\mathrm{Wu}$ (2001) within the DSX-formalism for applications to multiple stellar systems. In the DSX-framework, the hydrodynamic equations (energy equation and Euler equation) take the form

$$
\begin{aligned}
& \frac{\partial}{\partial T} \Sigma+\frac{\partial}{\partial X^{a}} \Sigma^{a}=\frac{1}{c^{2}} \frac{\partial}{\partial T} T^{b b}-\frac{1}{c^{2}} \Sigma \frac{\partial}{\partial T} W+O(4), \\
& \frac{\partial}{\partial T}\left[\left(1+\frac{4 W}{c^{2}}\right) \Sigma^{a}\right]+\frac{\partial}{\partial X^{b}}\left[\left(1+\frac{4 W}{c^{2}}\right) T^{a b}\right]=F^{a}\left(T, X^{a}\right)+O(4),
\end{aligned}
$$

where

$$
F^{a}=\Sigma E_{a}+\frac{1}{c^{2}} B_{a b} \Sigma^{b}, E_{a}=\partial_{a} W+\frac{4}{c^{2}} \partial_{T} W_{a}, B_{a b}=-4\left(\partial_{a} W_{b}-\partial_{b} W_{a}\right) .
$$

In the case of a non-perfect fluid the stress-energy tensor in local coordinates reads

$$
T^{\alpha \beta}=\epsilon U^{\alpha} U^{\beta}+p h^{\alpha \beta}-\frac{1}{3} \beta \theta h^{\alpha \beta}-\lambda \sigma^{\alpha \beta}+\frac{2}{c^{2}} Q^{(\mu} U^{\nu)},
$$

where $\epsilon$ is the density of total mass-energy, $p$ the isotropic pressure, $\lambda$ and $\beta$ are the coefficients of shear and bulk viscosity, $U^{\alpha}$ is the 4 -velocity, $h^{\alpha \beta}$ the usual projection operator into the fluid's rest space, $Q^{\alpha}$ the heat flux vector, $\sigma^{\alpha \beta}$ the shear tensor and $\theta=U_{; \mu}^{\mu}$ the expansion scalar. When $\lambda=\beta=\kappa=0, T^{\alpha \beta}$ describes a perfect fluid. $\epsilon$, $\theta, U^{\alpha}, h^{\alpha \beta}$ and $\sigma^{\alpha \beta}$ can then be explicitly expressed in terms of $\Sigma, \Sigma^{a}, W$ and $W^{a}$. $Q^{\alpha}$ also depends upon temperature $T$. If the heat flux is taken into account then $T^{\alpha \beta}$ depends upon $\Sigma, \Sigma^{a}, W, W^{a}, T, \lambda, \beta$ and $\kappa$. For reasons of brevity, here we only show the hydrodynamic equations for a perfect fluid

$$
\dot{\Sigma}+\Sigma_{, a}^{a}=\frac{1}{c^{2}}\left(\frac{2 \dot{\Sigma}^{d} \Sigma^{d}}{\Sigma}-\frac{\dot{\Sigma} \Sigma^{d} \Sigma^{d}}{\Sigma^{2}}+3 \dot{p}-\Sigma \dot{W}\right)+O(4),
$$




$$
\begin{aligned}
& \frac{\partial}{\partial T}\left[\left(1+\frac{4 W}{c^{2}}\right) \Sigma^{a}\right]+\frac{\partial}{\partial X^{b}}\left[( 1 + \frac { 4 W } { c ^ { 2 } } ) \left(\frac{\Sigma^{a} \Sigma^{b}}{\Sigma}\left(1+\frac{\Sigma^{c} \Sigma^{c}}{\Sigma^{2} c^{2}}+\frac{2 p}{\Sigma c^{2}}\right)\right.\right. \\
& \left.\left.\quad+p \delta^{a b}\left(1-\frac{2 W}{c^{2}}\right)\right)\right]=\Sigma \partial_{a} W+\frac{4}{c^{2}}\left[\Sigma \partial_{T} W_{a}-\Sigma^{b}\left(\partial_{a} W_{b}-\partial_{b} W_{a}\right)\right]+O(4) .
\end{aligned}
$$

The post-Newtonian expansion scalar reads

$$
\theta=U_{; \mu}^{\mu}=\frac{\partial V^{a}}{\partial X^{a}}+\frac{1}{c^{2}}\left[\frac{\partial V^{a}}{\partial X^{a}}\left(W+\frac{V^{2}}{2}\right)+3 \frac{d W}{d T}+\frac{1}{2} \frac{d}{d T} V^{2}\right]+O(4)
$$

Note that $\theta=0$ does not imply that $\partial V^{a} / \partial X^{a}=0$ (incompressible fluids only exists in Newtonian hydrodynamics, not in the general relativistic hydrodynamics). For more details the reader is referred to $\mathrm{Xu}$ and $\mathrm{Wu}$ (2001).

\section{Discussion}

Three different models for the dynamics of an astronomical body for an extension of the DSX-framework have been introduced: elastic bodies, fluid bodies and quasi-rigid bodies. Such models have been introduced in the local coordinate system that is comoving with the body under consideration and can be used in situations where multiple reference systems should be introduced. Several problems still have to be treated, e.g., for the problem of geodynamics an expansion of relevant functions in terms of generalized spherical harmonics has not yet been done for an oblate ground state, which is important for a relativistic theory of precession-nutation.

\section{Acknowledgements}

The authors thank the editor and the referee for improving the text and the English. This work was supported by the Natural Science Foundation of China under the grants 10673026 and 10873014.

\section{References}

Bretagnon, P., Rocher, P., \& Simon, J. L. 1997, A\&A, 319, 305

Carter, B. \& Quintana, H. 1972, Proc. Roy. Soc. London, A331, 57

Chandrasekhar, S. 1971, ApJ, 164, 569

Damour, T., Soffel, M., \& Xu, C. 1991, Phys. Rev. D 43, 3273

Damour, T., Soffel, M., \& Xu, C. 1992, Phys. Rev. D 45, 1017

Damour, T., Soffel, M., \& Xu, C. 1993, Phys. Rev. D 47, 3124

Damour, T., Soffel, M., \& Xu, C. 1994, Phys. Rev. D 49, 618

Jeffereys, H. \& Vicence, R. O. 1957, MNRAS, 117, 142

Soffel, M. 2009, this proceedings, 1

Tao, J. \& Xu, C. 2003, Int. J. Mod. Phys. D 12, 811

Wahr, J. M. 1982, Geo-dynamics, No. 41, 327

Xu, C. \& Wu, X. 2001, Phys. Rev. D 63, 064001

Xu, C., Wu, X., \& Soffel, M. 2001, Phys. Rev. D 63, 043002

Xu, C., Wu, X., Soffel, M., \& Klioner, S. 2003, Phys. Rev. D 68, 064009

Xu, C. \& Tao, J. 2004, Phys. Rev. D 69, 024003

Xu, C., Wu, X., \& Soffel, M. 2005, Phys. Rev. D 71, 024030 